\title{
Application of Dietary Fiber in Special Medicine
}

\author{
Jing Shi, Xiaofeng Shi, Yonghong Ma \\ Department of Public Health, Xi'an Medical University, Xi'an, Shaanxi, 710021
}

Keywords: Dietary Fiber, Special Medicine, Application Study

\begin{abstract}
Special medical use of formula food is to meet the dietary restriction, digestion and absorption disorders or metabolic disorders such as the daily nutritional needs of the crowd, or in order to meet certain medical conditions or disease groups of some nutrients or dietary special needs, specially formulated Of the food, these foods must be under the guidance of a doctor or clinical nutritionist, can be used alone, can also be used with ordinary food or other special dietary foods.
\end{abstract}

\section{Introduction}

Foods for special medical purposes (FSMP) is a type of specially formulated food formulated to meet the specific requirements for nutrients or diets in people with restricted diet, digestion and absorption disorders, metabolic disorders or specific disease states, Such food must be under the guidance of a doctor or clinical nutritionist, alone or in conjunction with other food consumption. FSMP is a special diet, and health food products without affiliation, may not claim health functions. In 2008, the Department of Medical Affairs of the Ministry of Health issued the "Opinions of the Ministry of Health on Strengthening Clinical Nutrition Work". FSMP has been rapidly developed. Its proportion in clinical nutrition has been increasing year by year and has been growing rapidly. The growth rate in 2008-2012 surpassed that of FSMP. 10\%, indicating that FSMP is increasingly clinically valued. In recent years, China has promulgated GB13432-2013 "pre-packaged food labels with special diet", GB2992-2013 "General Principles of Food Safety National Formulas for special medical uses," GB29923-2013 "national food safety standards for special medical uses good food Production Code "3 national standards. According to Order No. 24 of the State Food and Drug Administration, July 1, 2016, the Measures for the Administration of Registration of Formulations for Special Medical Purposes began. The FSMP industry is booming in the world. China has a large population, an increasing proportion of the elderly, and a strong demand for such products. FSMP provides nutritional support and can improve the prognosis of disease treatment in special populations such as the elderly, children and pregnant women. It is foreseeable that China's clinical and specific population will have a substantial increase in demand for whole nutrient fluid food, and its market prospect is broad. After many years of development, the variety of formula and composition of the prescriptions for special medical uses both at home and abroad have not been systematically analyzed and compared for different types of product formulations. Based on the research and application status of FSMP at home and abroad, In order to find out the characteristics of the different types of FSMP products, provide reference and reference for the research and development of FSMP in our country.

\section{Special Medical Use Food Importance}

Special medical uses of food for the patient is of great significance. Many inpatient surgery patients, will focus on the surgeon and surgeon doctors, often overlook the preoperative preparation and postoperative care, especially clinical nutrition. In fact, in many cases, special medical use foods have important clinical significance for patients. Special medical use foods can shorten the metabolic imbalance, reduce the complications such as infection, enhance the effect of various treatments and promote the rehabilitation. Hospital stay, improve patient quality of life and other aspects of important clinical significance. Special medical uses of food is also one of the means to 
reduce medical costs. The Brazilian Ministry of Health has conducted a health economics assessment. As a result of the assessment, the patient can spend 8 bucks for every 1 dollar they spend on nutrition support.

\section{Intestinal health is important for special dietary populations}

Intestinal tract is the body's most important digestive and immune organs and immune organs, but also one of the most important organs of the human body. At present, people are fully aware of the importance of the intestine on the basis of a new one, and put forward that whether the gastrointestinal tract function is intact or not is the gold standard for selecting enteral nutrition preparations. Therefore, in determining the patient's nutritional approach, intestinal function should be preferred in the enteral nutrition, and should try their best to implement, it has become the consensus of many clinicians.

Normal intestinal flora plays an important role in the construction, maintenance and destruction of gut barrier, which is closely related to the balance of gut microflora. Therefore, maintaining the integrity of intestinal barrier function from the gut microbiological point of view has important clinical significance: rational use of antibiotics to protect the fungal barrier function; to reduce bacterial translocation and clinical infection; to supplement medical probiotics, to help restore the intestine Road microecological balance regulation of immune function.

In the past 20 years, dietary fiber has been clinically regarded as one of the most important enteral nutrition components and has been widely used clinically. In recent years, clinical enteral nutrition treatment more and more choose to contain dietary fiber formula. By 2006, with the dietary fiber enteral nutrition formula has been as high as $60 \%$, and showed an upward trend year by year. Dietary fiber can be divided into soluble and insoluble dietary fiber, insoluble fiber can increase the volume of manure, accelerate intestinal peristalsis; soluble fiber can delay gastric emptying. Some soluble dietary fiber has the prebiotic effect, such as polydextrose, in addition to having insoluble dietary fiber unique to enhance laxatives, reduce the risk of intestinal cancer and other functions, but also with insoluble dietary fiber does not have or not obvious physiological function, Such as low energy; non-insulin-dependent, can significantly reduce postprandial blood glucose levels; lower triglycerides and significantly lower serum cholesterol; bifidobacteria have significant intestinal stimulation, in addition to Bifidobacterium adolescentis, Bifidobacterium longum Other proliferative effects, but also on the proliferation of infant Bifidobacteria. Polydextrose can reduce the rate of glycolysis, so that regulation of the role of the end to the colon, significantly maintaining the intestinal microflora balance. As a prebiotics, fructooligosaccharides have the same characteristics as water-soluble dietary fiber and are currently widely used in food development for special medical uses. Fructooligosaccharides in the large intestine, to promote the reproduction of a small number of beneficial bacteria such as Bifidobacteria role, and can significantly inhibit the reproduction of harmful bacteria, improve intestinal microflora and promote gastrointestinal health; the same time, with improved lipid metabolism, reduce Lipid and cholesterol, improve immunity, inhibition, prevention of cancer and promote the absorption of calcium and magnesium mineral elements, which are the physiological role of insoluble dietary fiber can not be replaced, its development in the special medical use of food, clinical intestine Internal nutrition therapy plays an important role.

In 1990, researchers in animal experiments showed that the positive rates of mesenteric lymph node bacterial culture in total parenteral nutrition and single pectin group were significantly higher than those in dietary fiber group, while those in cellulose group, corn and Kaolin (including dietary fiber) were not What changed, confirming that enteral nutrition with dietary fiber helps to maintain the gut barrier and prevent bacterial translocation. Non-fiber foods can promote bacterial translocation, while the dietary fiber can maintain the normal ecological balance of intestinal flora. The bacterial metabolic fiber end products have a nutritious effect on the small intestine epithelium, and the fiber can maintain the normal metabolism and cell kinetics of intestinal mucosal cells. Therefore, adding a certain proportion of fiber components to the daily diet or the nutritional formula of the patient can maintain the normal proportion of the intestinal flora of the patient, 
inhibit the growth of pathogenic bacteria, prevent the intestinal mucosa from atrophy and reduce the occurrence of intestinal bacterial translocation .

\section{Dietary Fiber Market Products and Applications}

Huarui Rui can: a high fat, high energy, low carbohydrate content of enteral nutrition, especially for cancer patients metabolic needs, of which $18 \%$ protein, $50 \%$ fat, carbohydrates $32 \%$, dietary fiber $1.3 \mathrm{~g} / 100 \mathrm{~m} \mathrm{~L}$, dietary fiber helps to maintain gastrointestinal function. Huarui Ruixian: can be used as a source of all nutrients or nutritional supplements to patients who can not eat normally, especially those who can not tolerate high-volume feeding or high energy patients, rich in dietary fiber, is conducive to maintaining the patient's intestinal structure And function, suitable for long-term application. Huarui Rui Dai: Diabetes and stress-induced hyperglycemic enteral nutrition preparations, high-dietary fiber formulations, help to stabilize the patient's blood glucose levels, to maintain gastrointestinal function. Can be full: dietary fiber: $2.2 \mathrm{~g} / 100 \mathrm{~mL}$, for patients with limited fluid intake, cardiopulmonary insufficiency, renal insufficiency, traumatic brain injury, cerebral edema, in a highly metabolic state of patients, severe infections, trauma and burns Other stress conditions. Abbott Good Glucerna SR: 20\% protein, 32.6\% fat, 47.5\% carbohydrates, rich in MFA, low electrolyte, dietary fiber $0.76 \mathrm{~g} / 100 \mathrm{~m}$ L. Li Shikang homogenate (fiber type): balanced, nutritionally homogenized formula, high protein content, add Gln, high dietary fiber content. Nestle diet fiber: dietary fiber with a scientific combination to promote intestinal health, either as a critical source of nutrition only to meet the daily nutritional needs of all, to help their rapid recovery; can also be used as a dietary supplement to daily diet and enhance physical strength .

Since the 90s of last century in our country, socio-economic growth and material civilization have developed. Some "civilized diseases" - all kinds of malignancies, hyperlipidemia, hypertension, arteriosclerosis, diabetes and obesity are on the rise. With everyone's health. The formation of an aging society, a fast-paced life style, the existence of a large number of sub-health groups, as well as a variety of specific physiological stages of the population, have special needs of food nutrition. This has given a special mission food special diet, will also stimulate the development of special medical uses of food in our country. (1) the use of nutrients to achieve the role of drug therapy, not just the role of nutrition. (2) Preventive nutrition support, which means nourishing support before elective surgery. (3) Develop specific and scientific formulas for patients with specific diseases, which are scientific and reasonable, meet the daily nutritional needs of the target population, and can meet the special medical needs of certain nutrients due to specific diseases, metabolic disorders and medical conditions. (4) develop diabetes-specific food. That is strictly controlled by heat, rich in high-quality protein and carbohydrates, low fat, high fiber, non-low molecular sugars, high cellulose and minerals, active substances in food. (5) Special diet for special populations at specific physiologic stages is one of the main development directions. Such as suitable for the elderly to eat malnutrition food: rich in dietary fiber, protein, mineral elements, vitamins, low calorie, low fat, low cholesterol, low sodium salt foods; suitable for children's growth and development of food; suitable for pregnancy, lactation Food

\section{Conclusion}

With the economic growth and improvement of people's living standards and special medical use of food and nutrition treatment gradually accepted by special diet people, the most important is the special medical use of formula-related laws and regulations and the relevant details of the introduction, production, circulation, Regulation will be law-abiding, and the next few years will be an important period for the rapid development of special medical-use foods in China.

\section{References}

[1] Márta K, Farkas N, Szabó I, et al. Meta-Analysis of Early Nutrition: The Benefits of Enteral Feeding Compared to a Nil Per Os Diet Not Only in Severe, but Also in 
Mild and Moderate Acute Pancreatitis[J]. Int J Mol Sci. 2016 17(10):1691-1693.

[2] Stameneković D, Basić M, Palibrk I,et al. Enteral nutrition and surgical patient[J].Acta Chir Iugosl., 2003;50(4):109-113.

[3] Guo GH, Deng ZY, Wang YX,et al. Effects of glutamine enriched enteral feeding on immunoregulation in burn patients[J].Zhonghua Shao Shang Za Zhi., 2007,23(6):406-408.

[4] Paccagnella A, Baruffi C, Pizzolato D, et al. Home enteral nutrition in adults: A five-year (2001-2005) epidemiological analysis[J]. Clin Nutr. , 2008; 27:378-385.

[5] Moyes LH, Mc Kee RF. A review of surgical nutrition [J]. Scott Med J. 2008, 53:38-43.

[6] Pugaev AV, Lidov PI, Achkasov EE, et al. Enteral nutrition in the complex treatment of patients with mechanical jaundice in gallstone disease[J].Klin Med (Mosk)., 2008;86:51-53.

[7] Jiang W. Early enteral nutrition for upper digestive tract malformation in neonate [J]. Asia Pac J Clin Nutr. 